\title{
Cationic Rhodium(I)/BINAP Complex-Catalyzed Isomerization of Secondary Propargylic Alcohols to $\alpha, \beta$-Enones
}

\author{
Ken Tanaka* and Takeaki Shoji \\ Department of Applied Chemistry, Graduate School of Engineering, Tokyo University \\ of Agriculture and Technology, Koganei, Tokyo 184-8588, Japan

\section{General}

Anhydrous $\mathrm{CH}_{2} \mathrm{Cl}_{2}$ (No. 27,099-7), anhydrous $\left(\mathrm{CH}_{2} \mathrm{Cl}\right)_{2}$ (No. 28,450-5), and anhydrous acetone (No. 27,072-5) were obtained from Aldrich and used as received. Tol-BINAP, H8-BINAP, and Segphos were obtained from Takasago International Corporation. All other reagents were obtained from commercial sources and used as received. All reactions were carried out under an atmosphere of argon in oven-dried glassware with magnetic stirring, unless otherwise indicated.

\section{Synthesis of Propargylic Alcohols}

\section{1-Phenylpent-1-yn-3-ol (1a). ${ }^{1}$}

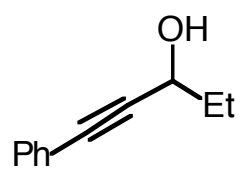

$n$-BuLi (1.58 M in hexane, $11.5 \mathrm{~mL}, 18.2 \mathrm{mmol})$ was added to a stirred solution of phenylacetylene $(1.9 \mathrm{~g}, 18.2 \mathrm{mmol})$ in THF $(20 \mathrm{~mL})$ at $0{ }^{\circ} \mathrm{C}$, and the mixture was stirred at $0{ }^{\circ} \mathrm{C}$ for $30 \mathrm{~min}$. To the resulting solution was added propionaldehyde $(1.3 \mathrm{~mL}, 18.2$ $\mathrm{mmol})$ at $0{ }^{\circ} \mathrm{C}$, and the mixture was gradually warmed to $\mathrm{rt}$. The reaction was quenched by the addition of water and extracted with $\mathrm{Et}_{2} \mathrm{O}$. The organic layer was washed with brine, dried over $\mathrm{Na}_{2} \mathrm{SO}_{4}$, and concentrated. The residue was purified by silica gel column chromatography (hexane:EtOAc $=8: 1$ ), which furnished 1-phenylpent-1-yn-3ol $1 \mathbf{a}(2.7 \mathrm{~g}, 16.6 \mathrm{mmol}, 92 \%$ yield $)$ as a pale yellow oil.

${ }^{1} \mathrm{H}$ NMR $\left(\mathrm{CDCl}_{3}, 400 \mathrm{MHz}\right) \delta 7.37-7.46(\mathrm{~m}, 2 \mathrm{H}), 7.26-7.37(\mathrm{~m}, 3 \mathrm{H}), 4.50-4.60(\mathrm{~m}$, $1 \mathrm{H}), 1.98-2.05(\mathrm{~m}, 1 \mathrm{H}), 1.75-1.88(\mathrm{~m}, 2 \mathrm{H}), 1.08(\mathrm{t}, J=7.6 \mathrm{~Hz}, 3 \mathrm{H}) ;{ }^{13} \mathrm{C} \mathrm{NMR}\left(\mathrm{CDCl}_{3}\right.$, $75 \mathrm{MHz}) \delta 131.7,128.3,128.2,122.6,89.9,84.9,64.2,30.9,9.5$.

\section{4-Phenylbut-3-yn-2-ol (1b). ${ }^{2}$}

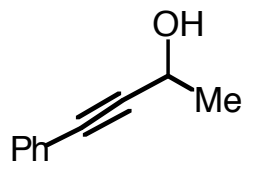

The title compound was prepared as a yellow oil in $67 \%$ isolated yield from acetaldehyde according to the procedure of $\mathbf{1 a}$.

${ }^{1} \mathrm{H}$ NMR $\left(\mathrm{CDCl}_{3}, 300 \mathrm{MHz}\right) \delta 7.35-7.50(\mathrm{~m}, 2 \mathrm{H}), 7.25-7.40(\mathrm{~m}, 3 \mathrm{H}), 4.70-4.85(\mathrm{~m}$, 
$1 \mathrm{H}), 1.90(\mathrm{~d}, J=5.4 \mathrm{~Hz} 1 \mathrm{H}) 1.55(\mathrm{~d}, J=6.6 \mathrm{~Hz}, 3 \mathrm{H}) ;{ }^{13} \mathrm{C} \mathrm{NMR}\left(\mathrm{CDCl}_{3}, 75 \mathrm{MHz}\right) \delta$ $131.6,128.3,128.2,122.5,90.9,83.9,58.8,24.3$.

\section{1-Phenylhept-1-yn-3-ol (1c). ${ }^{1}$}<smiles>CCCCCC(O)C#Cc1ccccc1</smiles>

The title compound was prepared as a colorless solid in $91 \%$ isolated yield from valeraldehyde according to the procedure of $\mathbf{1 a}$.

${ }^{1} \mathrm{H}$ NMR $\left(\mathrm{CDCl}_{3}, 300 \mathrm{MHz}\right) \delta 7.38-7.48(\mathrm{~m}, 2 \mathrm{H}), 7.24-7.36(\mathrm{~m}, 3 \mathrm{H}), 4.60(\mathrm{q}, J=6,3$ $\mathrm{Hz}, 1 \mathrm{H}), 1.90-2.00(\mathrm{~m}, 1 \mathrm{H}) 1.70-1.88(\mathrm{~m}, 2 \mathrm{H}), 1.30-1.58(\mathrm{~m}, 4 \mathrm{H}), 0.94(\mathrm{t}, J=7.2 \mathrm{~Hz}$, $3 \mathrm{H}) ;{ }^{13} \mathrm{C} \mathrm{NMR}\left(\mathrm{CDCl}_{3}, 75 \mathrm{MHz}\right) \delta 131.6,128.3,128.2,122.6,90.2,84.7,62.9,37.6$, $27.3,22.4,14.0$.

\section{4-Methyl-1-phenylpent-1-yn-3-ol (1d). ${ }^{3}$}<smiles>CCCC(O)C#Cc1ccccc1</smiles>

The title compound was obtained from Lancaster.

${ }^{1} \mathrm{H}$ NMR $\left(\mathrm{CDCl}_{3}, 300 \mathrm{MHz}\right) \delta$ 7.39-7.48 (m, 2H), 7.25-7.36 (m, 3H), 4.40 (d, $J=6.6$ $\mathrm{Hz}, 1 \mathrm{H}), 1.80-2.07(\mathrm{~m}, 2 \mathrm{H}), 1.07(\mathrm{t}, J=6.6 \mathrm{~Hz}, 6 \mathrm{H}) ;{ }^{13} \mathrm{C} \mathrm{NMR}\left(\mathrm{CDCl}_{3}, 75 \mathrm{MHz}\right) \delta$ $131.7,128.3,128.2,122.7,88.9,85.5,68.3,34.7,18.2,17.5$.

\section{4, 4-Dimetyl-1-phenylpent-1-yn-3-ol (1e). ${ }^{3}$}<smiles>CC(C)(C)C(O)C#Cc1ccccc1</smiles>

The title compound was prepared as a colorless solid in $99 \%$ isolated yield from trimethylacetaldehyde according to the procedure of $\mathbf{1 a}$.

${ }^{1} \mathrm{H} \mathrm{NMR}\left(\mathrm{CDCl}_{3}, 300 \mathrm{MHz}\right) \delta 7.38-7.48(\mathrm{~m}, 2 \mathrm{H}), 7.24-7.36(\mathrm{~m}, 3 \mathrm{H}), 4.23(\mathrm{~d}, J=6.3$ $\mathrm{Hz}, 1 \mathrm{H}), 1.86(\mathrm{~d}, J=6.3 \mathrm{~Hz}, 1 \mathrm{H}) 1.07(\mathrm{~s}, 9 \mathrm{H}) ;{ }^{13} \mathrm{C} \mathrm{NMR}\left(\mathrm{CDCl}_{3}, 75 \mathrm{MHz}\right) \delta 131.6$, $128.22,128.20,122.7,88.9,85.6,71.7,36.0,25.3$.

\section{1-o-Tolylpent-1-yn-3-ol (1h).}<smiles>CCC(O)C#Cc1ccccc1C</smiles> 
A solution of $\mathrm{CBr}_{4}(22.2 \mathrm{~g}, 66.9 \mathrm{mmol})$ in $\mathrm{CH}_{2} \mathrm{Cl}_{2}(50 \mathrm{~mL})$ was added dropwise to a solution of $\mathrm{PPh}_{3}(35.1 \mathrm{~g}, 133.8 \mathrm{mmol})$ in $\mathrm{CH}_{2} \mathrm{Cl}_{2}(50 \mathrm{~mL})$ at $0{ }^{\circ} \mathrm{C}$. After stirring at $0{ }^{\circ} \mathrm{C}$ for $5 \mathrm{~min}$, a solution of 2-methylbenzaldehyde $(4.2 \mathrm{~g}, 33.5 \mathrm{mmol})$ was added dropwise to the reaction mixture at $0{ }^{\circ} \mathrm{C}$. The resulting mixture was stirred at $0{ }^{\circ} \mathrm{C}$ for $20 \mathrm{~min}$. The reaction was quenched by the addition of water and saturated aqueous $\mathrm{NaHCO}_{3}$. The resulting mixture was extracted with $\mathrm{CH}_{2} \mathrm{Cl}_{2}$, dried over $\mathrm{Na}_{2} \mathrm{SO}_{4}$, and concentrated. The residue was purified by silica gel chromatography (hexane:EtOAc $=8: 1$ ), which furnished 1-(2,2-dibromovinyl)-2-methylbenzene (7.3 g, $26.6 \mathrm{mmol}, 79 \%$ yield) as a pale yellow solid.

$n$-BuLi (1.58 $\mathrm{M}$ in hexane, $9.1 \mathrm{~mL}, 14.3 \mathrm{mmol})$ was added to a stirred solution of 1(2,2-dibromovinyl)-2-methylbenzene $(2.0 \mathrm{~g}, 7.2 \mathrm{mmol})$ in THF $(20 \mathrm{~mL})$ at $-10{ }^{\circ} \mathrm{C}$, and the resulting mixture was stirred at $-10{ }^{\circ} \mathrm{C}$ for $30 \mathrm{~min}$. To the resulting solution was added propionaldehyde $(0.50 \mathrm{~mL}, 7.2 \mathrm{mmol})$ at $-10^{\circ} \mathrm{C}$, and the mixture was gradually warmed to rt. The reaction was quenched by the addition of water and extracted with $\mathrm{Et}_{2} \mathrm{O}$. The organic layer was washed with brine, dried over $\mathrm{Na}_{2} \mathrm{SO}_{4}$, and concentrated. The residue was purified by silica gel column chromatography (hexane:EtOAc $=8: 1$ ), which furnished 1-o-tolylpent-1-yn-3-ol (1.2 g, $6.6 \mathrm{mmol}, 92 \%)$ as a pale yellow oil.

IR (neat) $3300,2950,1015,745 \mathrm{~cm}^{-1} ;{ }^{1} \mathrm{H}$ NMR $\left(\mathrm{CDCl}_{3}, 300 \mathrm{MHz}\right) \delta 7.37-7.42(\mathrm{~m}$, $1 \mathrm{H}), 7.09-7.25(\mathrm{~m}, 3 \mathrm{H}), 4.64(\mathrm{q}, J=6.0 \mathrm{~Hz}, 1 \mathrm{H}), 2.43(\mathrm{~s}, 3 \mathrm{H}), 1.93-2.00(\mathrm{~m}, 1 \mathrm{H})$, $1.78-1.90(\mathrm{~m}, 2 \mathrm{H}), 1.09(\mathrm{t}, J=7.5 \mathrm{~Hz}, 3 \mathrm{H}) ;{ }^{13} \mathrm{C} \mathrm{NMR}\left(\mathrm{CDCl}_{3}, 100 \mathrm{MHz}\right) \delta 140.0$, 131.8, 129.3, 128.3, 125.4, 122.3, 93.8, 83.7, 64.3, 31.1, 20.8, 9.6; HRMS (EI) calcd for $\mathrm{C}_{12} \mathrm{H}_{14} \mathrm{O}[\mathrm{M}]^{+}$174.1045, found 174.1011.

\section{1-(4-Methoxyphenyl)pent-1-yn-3-ol (1f).}

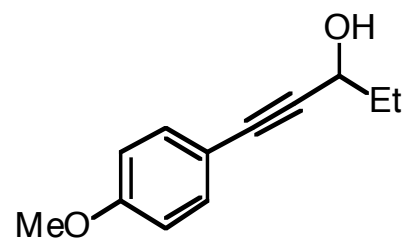

The title compound was prepared as a pale yellow oil in $89 \%$ isolated yield from 1(2,2-dibromovinyl)-4-methoxybenzene and propionaldehyde according to the procedure of $1 \mathbf{h}$.

IR (neat) 3350, 2950, 1600, 1508, 1245, 1180, 1030, $835 \mathrm{~cm}^{-1} ;{ }^{1} \mathrm{H}$ NMR $\left(\mathrm{CDCl}_{3}, 300\right.$ MHz) $\delta 7.34-7.40(\mathrm{~m}, 2 \mathrm{H}), 6.80-6.86(\mathrm{~m}, 2 \mathrm{H}), 4.54(\mathrm{q}, J=6.3 \mathrm{~Hz}, 1 \mathrm{H}), 3.80(\mathrm{~s}, 3 \mathrm{H})$, 1.96-2.06 (m, 1H), 1.74-1.90 (m, 2H), $1.07(\mathrm{t}, J=7.5 \mathrm{~Hz}, 3 \mathrm{H}) ;{ }^{13} \mathrm{C}$ NMR $\left(\mathrm{CDCl}_{3}, 75\right.$ MHz) $\delta 159.6,133.1,114.7,113.8,88.5,84.8,64.2,55.2,31.0,9.5$. HRMS (EI) calcd for $\mathrm{C}_{12} \mathrm{H}_{14} \mathrm{O}_{2}[\mathrm{M}]^{+}$190.0994, found 190.0970.

\section{1-(4-Trifluoromethylphenyl)pent-1-yn-3-ol (1g). ${ }^{4}$}




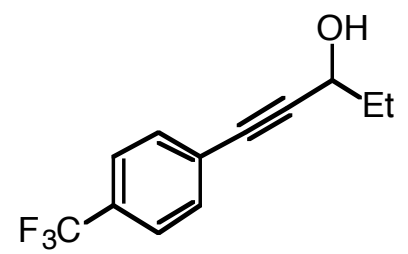

The title compound was prepared as a pale brown oil in 74\% isolated yield from 1(2,2-dibromovinyl)-4-trifluoromethylbenzene and propionaldehyde according to the procedure of $\mathbf{1 h}$.

${ }^{1} \mathrm{H} \mathrm{NMR}\left(\mathrm{CDCl}_{3}, 300 \mathrm{MHz}\right) \delta 7.50-7.61(\mathrm{~m}, 4 \mathrm{H}), 4.57(\mathrm{q}, J=6.0 \mathrm{~Hz}, 1 \mathrm{H}), 2.08(\mathrm{~d}, J$ $=5.7 \mathrm{~Hz}, 1 \mathrm{H}), 1.78-1.90(\mathrm{~m}, 2 \mathrm{H}), 1.09(\mathrm{t}, J=7.5 \mathrm{~Hz}, 3 \mathrm{H}) ;{ }^{13} \mathrm{C} \mathrm{NMR}\left(\mathrm{CDCl}_{3}, 75 \mathrm{MHz}\right)$ $\delta 131.9,130.3,129.9,126.4,125.6,125.2(\mathrm{q}), 122.0,92.4,83.6,64.1,30.8,9.5$.

\section{2-Deuterio-4-phenylbut-3-yn-2-ol (1b-d).}

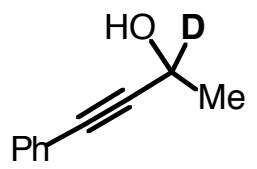

To a solution of 4-phenyl-3-butyn-2-ol $(\mathbf{1 b}, 1.0 \mathrm{~g}, 6.8 \mathrm{mmol})$ in acetone $(20 \mathrm{~mL})$ was added Jones reagent $(1.5 \mathrm{M}, 5.1 \mathrm{~mL}, 7.6 \mathrm{mmol})$ at $0{ }^{\circ} \mathrm{C}$. After stirring at $0{ }^{\circ} \mathrm{C}$ for 15 $\mathrm{min}$, the reaction was quenched by the addition of $2-\mathrm{PrOH}(6.2 \mathrm{~mL})$ at $0{ }^{\circ} \mathrm{C}$. The mixture was stirred at $\mathrm{rt}$ for $10 \mathrm{~min}$ and concentrated. The residue was diluted with water and extracted with $\mathrm{Et}_{2} \mathrm{O}$. The organic layer was concentrated and purified by silica gel chromatography (hexane:EtOAc $=6: 1$ ), which furnished 4-phenyl-3-butyn-2-on (0.49 g, $3.4 \mathrm{mmol}, 50 \%$ yield) as a colorless oil.

To a stirred mixture of $\mathrm{NaBD}_{4}(70.0 \mathrm{mg}, 1.7 \mathrm{mmol})$ in EtOH $(20 \mathrm{~mL})$ was added a $\mathrm{EtOH}(5.0 \mathrm{~mL})$ solution of 4-phenylbut-3-yn-2-on $(0.49 \mathrm{~g}, 3.4 \mathrm{mmol})$ at $0{ }^{\circ} \mathrm{C}$. The resulting mixture was stirred at $0{ }^{\circ} \mathrm{C}$ for $1 \mathrm{~h}$. The reaction was quenched with aqueous $\mathrm{HCl}(2 \mathrm{M}, 10 \mathrm{~mL})$, diluted with brine $(80 \mathrm{~mL})$, and extracted with $\mathrm{CH}_{2} \mathrm{Cl}_{2}$. The organic layer was concentrated and purified by silica gel chromatography (hexane:EtOAc $=$ 6:1), which furnished 2-deuterio-4-phenylbut-3-yn-2-ol (1b- $d, 0.40 \mathrm{~g}, 2.7 \mathrm{mmol}, 81 \%$ yield) as a colorless oil.

${ }^{1} \mathrm{H}$ NMR $\left(\mathrm{CDCl}_{3}, 300 \mathrm{MHz}\right) \delta$ 7.40-7.46 (m, 2H), 7.27-7.35 (m, 3H), 1.96-2.05 (m, $1 \mathrm{H}), 1.55(\mathrm{~s}, 3 \mathrm{H}) ;{ }^{2} \mathrm{H} \mathrm{NMR}\left(\mathrm{CDCl}_{3}, 61 \mathrm{MHz}\right) \delta 4.76(\mathrm{~s}, 1 \mathrm{H})$.

\section{Isomerization of Secondary Propargylic Alcohols}

General Procedure 1 (Table 2, entry 1). Under an $\mathrm{Ar}$ atmosphere, a $\mathrm{CH}_{2} \mathrm{Cl}_{2}(0.5$ $\mathrm{mL})$ solution of BINAP $(15.6 \mathrm{mg}, 0.025 \mathrm{mmol})$ was added to a $\mathrm{CH}_{2} \mathrm{Cl}_{2}(0.5 \mathrm{~mL})$ solution of $\left[\mathrm{Rh}(\operatorname{cod})_{2}\right] \mathrm{BF}_{4}(10.2 \mathrm{mg}, 0.025 \mathrm{mmol})$ at $\mathrm{rt}$, and the mixture was stirred for 5 min at $\mathrm{rt} . \mathrm{H}_{2}$ was introduced to the resulting solution in a Schlenk tube. After stirring for $0.5 \mathrm{~h}$ at $\mathrm{rt}$, the resulting solution was concentrated to dryness and dissolved in $\left(\mathrm{CH}_{2} \mathrm{Cl}\right)_{2}$ $(1.0 \mathrm{~mL})$. To this solution was added a $\left(\mathrm{CH}_{2} \mathrm{Cl}\right)_{2}(0.5 \mathrm{~mL})$ solution of 4-phenylbut-3-yn2-ol (1b, $73.1 \mathrm{mg}, 0.5 \mathrm{mmol})$, and washed remaining substrate away by using $\left(\mathrm{CH}_{2} \mathrm{Cl}\right)_{2}$ $(0.5 \mathrm{~mL})$. The solution was stirred at $80{ }^{\circ} \mathrm{C}$ for $1 \mathrm{~h}$. The resulting solution was 
concentrated and purified by silica gel column chromatography $\left(\mathrm{Et}_{2} \mathrm{O}\right)$ to give 4phenylbut-3-en-2-one (2b, $72.5 \mathrm{mg}, 0.495 \mathrm{mmol}, 99 \%$ yield, $E: Z=100: 0)$ as an orange solid.

\section{(E)-4-Phenylbut-3-en-2-one (2b). ${ }^{5}$}<smiles>CC(=O)/C=C/c1ccccc1</smiles>

Mp 35-37 ${ }^{\circ} \mathrm{C} ;{ }^{1} \mathrm{H}$ NMR $\left(\mathrm{CDCl}_{3}, 300 \mathrm{MHz}\right) \delta 7.48-7.60(\mathrm{~m}, 3 \mathrm{H}), 7.30-7.48(\mathrm{~m}, 3 \mathrm{H})$, $6.72(\mathrm{~d}, J=15.9 \mathrm{~Hz}, 1 \mathrm{H}), 2.37(\mathrm{~s}, 3 \mathrm{H}) ;{ }^{13} \mathrm{C} \mathrm{NMR}\left(\mathrm{CDCl}_{3}, 75 \mathrm{MHz}\right) \delta 198.4,143.4$, $134.3,130.5,128.9,128.2,127.1,27.5$.

\section{(E)-1-Phenylpent-1-en-3-one (2a, $E: Z=100: 0){ }^{6}$}

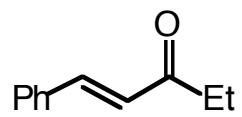

Orange oil; ${ }^{1} \mathrm{H}$ NMR $\left(\mathrm{CDCl}_{3}, 300 \mathrm{MHz}\right) \delta 7.50-7.62(\mathrm{~m}, 3 \mathrm{H}), 7.26-7.50(\mathrm{~m}, 3 \mathrm{H})$, $6.75(\mathrm{~d}, J=16.2 \mathrm{~Hz}, 1 \mathrm{H}), 2.71(\mathrm{q}, J=7.2 \mathrm{~Hz}, 2 \mathrm{H}) 1.17(\mathrm{t}, J=7.2 \mathrm{~Hz}, 3 \mathrm{H}) ;{ }^{13} \mathrm{C} \mathrm{NMR}$ $\left(\mathrm{CDCl}_{3}, 75 \mathrm{MHz}\right) \delta 200.9,142.2,134.5,130.3,128.9,128.2,126.0,34.0,8.2$.

(E)-1-Phenylhept-1-en-3-one (2c, $E: Z=100: 0)^{7}$<smiles>CCCCC(=O)C=Cc1ccccc1</smiles>

Pale yellow solid; Mp 32-33 ${ }^{\circ} \mathrm{C} ;{ }^{1} \mathrm{H}$ NMR $\left(\mathrm{CDCl}_{3}, 300 \mathrm{MHz}\right) \delta 7.50-7.60(\mathrm{~m}, 3 \mathrm{H})$, $7.30-7.50(\mathrm{~m}, 3 \mathrm{H}), 6.75(\mathrm{~d}, J=15.9 \mathrm{~Hz}, 1 \mathrm{H}), 2.67(\mathrm{t}, J=7.2 \mathrm{~Hz}, 2 \mathrm{H}), 1.60-1.74(\mathrm{~m}$, $2 \mathrm{H}), 1.30-1.47(\mathrm{~m}, 2 \mathrm{H}), 0.92(\mathrm{t}, J=7.2 \mathrm{~Hz}, 3 \mathrm{H}) ;{ }^{13} \mathrm{C} \mathrm{NMR}\left(\mathrm{CDCl}_{3}, 75 \mathrm{MHz}\right) \delta 200.5$, $142.2,134.5,130.3,128.8,128.1,126.1,40.7,26.5,22.5,14.0$.

(E)-4-Methyl-1-phenylpent-1-en-3-one (2d, $E: Z=100: 0){ }^{8}$

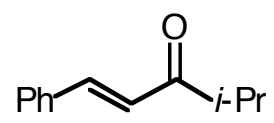

Orange oil; ${ }^{1} \mathrm{H}$ NMR $\left(\mathrm{CDCl}_{3}, 300 \mathrm{MHz}\right) \delta 7.50-7.60(\mathrm{~m}, 3 \mathrm{H}), 7.30-7.50(\mathrm{~m}, 3 \mathrm{H})$, $6.82(\mathrm{~d}, J=16.2 \mathrm{~Hz}, 1 \mathrm{H}), 2.94$ (septet, $J=6.9 \mathrm{~Hz}, 1 \mathrm{H}) 1.19(\mathrm{~d}, J=6.9 \mathrm{~Hz}, 6 \mathrm{H}) ;{ }^{13} \mathrm{C}$ NMR $\left(\mathrm{CDCl}_{3}, 75 \mathrm{MHz}\right) \delta 203.7,153.7,142.3,134.6,130.3,128.8,128.2,124.4,114.5$, $55.6,39.2,18.4$. 
(E)-4, 4-Dimethyl-1-phenylpent-1-en-3-one (2e, $E: Z=100: 0){ }^{9}$<smiles>CC(C)(C)C(=O)/C=C/c1ccccc1</smiles>

Pale yellow oil; ${ }^{1} \mathrm{H}$ NMR $\left(\mathrm{CDCl}_{3}, 300 \mathrm{MHz}\right) \delta 7.69(\mathrm{~d}, J=15.6 \mathrm{~Hz}, 1 \mathrm{H}), 7.50-7.65$ $(\mathrm{m}, 2 \mathrm{H}), 7.34-7.50(\mathrm{~m}, 3 \mathrm{H}), 7.13(\mathrm{~d}, J=15.6 \mathrm{~Hz}, 1 \mathrm{H}), 1.20(\mathrm{~s}, 9 \mathrm{H}) ;{ }^{13} \mathrm{C}$ NMR $\left(\mathrm{CDCl}_{3}\right.$, $75 \mathrm{MHz}) \delta 204.1,142.8,134.8,130.1,128.8,128.2,120.6,43.2,26.2$.

(E)-1-(4-Methoxyphenyl)pent-1-en-3-one $(2 \mathrm{f}, E: Z=100: 0){ }^{10}$<smiles>CCC(=O)/C=C/c1ccc(OC)cc1</smiles>

Orange solid; Mp 47-49 ${ }^{\circ} \mathrm{C} ;{ }^{1} \mathrm{H}$ NMR $\left(\mathrm{CDCl}_{3}, 400 \mathrm{MHz}\right) \delta 7.44-7.60(\mathrm{~m}, 3 \mathrm{H})$, 6.85-6.95 (m, 2H), $6.63(\mathrm{~d}, J=16.0 \mathrm{~Hz}, 1 \mathrm{H}), 3.84(\mathrm{~s}, 3 \mathrm{H}), 2.68(\mathrm{q}, J=7.2 \mathrm{~Hz}, 2 \mathrm{H})$, $1.16(\mathrm{t}, J=7.2 \mathrm{~Hz}, 3 \mathrm{H}) ;{ }^{13} \mathrm{C} \mathrm{NMR}\left(\mathrm{CDCl}_{3}, 100 \mathrm{MHz}\right) \delta 200.7,161.3,141.8,129.7$, 127.0, 123.7, 114.2, 55.3, 33.9, 8.4.

(E)-1-(4-Trifluoromethylphenyl)pent-1-en-3-one (2g, $E: Z=100: 0)$.<smiles>CCC(=O)/C=C/c1ccc(C(F)(F)F)cc1</smiles>

Pale brown solid; Mp 62-64 ${ }^{\circ} \mathrm{C}$; IR (neat) 2950, 1660, 1320, 1160, 1120, 985, 840 $\mathrm{cm}^{-1} ;{ }^{1} \mathrm{H}$ NMR $\left(\mathrm{CDCl}_{3}, 300 \mathrm{MHz}\right) \delta 7.65(\mathrm{~s}, 4 \mathrm{H}), 7.57(\mathrm{~d}, J=16.2 \mathrm{~Hz}, 1 \mathrm{H}), 6.81(\mathrm{~d}, J=$ $16.2 \mathrm{~Hz}, 1 \mathrm{H}), 2.72(\mathrm{q}, J=7.5 \mathrm{~Hz} 2 \mathrm{H}), 1.18(\mathrm{t}, J=7.5 \mathrm{~Hz}, 3 \mathrm{H}) ;{ }^{13} \mathrm{C}$ NMR $\left(\mathrm{CDCl}_{3}, 100\right.$ MHz) $\delta 200.2,140.0,137.9,131.8,128.2,127.9,125.7$ (q), 122.3, 34.4, 8.1; HRMS (EI) calcd for $\mathrm{C}_{12} \mathrm{H}_{11} \mathrm{~F}_{3} \mathrm{O}[\mathrm{M}]^{+} 228.0762$, found 228.0736.

(E)-1-o-Tolylpent-1-en-3-one $(2 \mathrm{~h}, E: Z=67: 33)$.<smiles>CCC(=O)/C=C/c1ccccc1C</smiles>

Orange oil; IR (neat) 2925, 1660, 1600, 1120, 1040, 980, $745 \mathrm{~cm}^{-1} ;{ }^{1} \mathrm{H}$ NMR $\left(\mathrm{CDCl}_{3}\right.$, $400 \mathrm{MHz}) E$ isomer: $\delta 7.86(\mathrm{~d}, J=15.6 \mathrm{~Hz}, 1 \mathrm{H}), 7.54-7.60(\mathrm{~m}, 1 \mathrm{H}), 7.10-7.37(\mathrm{~m}, 3 \mathrm{H})$, $6.67(\mathrm{~d}, J=15.6 \mathrm{~Hz}, 1 \mathrm{H}), 2.70(\mathrm{q}, J=7.2 \mathrm{~Hz}, 2 \mathrm{H}), 2.25(\mathrm{~s}, 3 \mathrm{H}), 1.18(\mathrm{t}, J=7.2 \mathrm{~Hz}$, 3H); $Z$ isomer: $7.10-7.37(\mathrm{~m}, 4 \mathrm{H}), 7.05(\mathrm{~d}, J=12.4 \mathrm{~Hz}, 1 \mathrm{H}), 6.22(\mathrm{~d}, J=12.4 \mathrm{~Hz}, 1 \mathrm{H})$, $2.30(\mathrm{~s}, 3 \mathrm{H}), 2.30(\mathrm{q}, J=7.2 \mathrm{~Hz}, 2 \mathrm{H}), 0.96(\mathrm{t}, J=7.2 \mathrm{~Hz}, 3 \mathrm{H}) ;{ }^{13} \mathrm{C} \mathrm{NMR}\left(\mathrm{CDCl}_{3}, 100\right.$ 
MHz) $\delta 203.8,200.7,139.5,139.1,137.7,135.6,135.3,133.4,130.7,129.9,129.8$, 129.7, 128.8, 128.5, 126.8, 126.2, 126.1, 125.5, 36.1, 34.3, 19.9, 19.8, 8.3, 8.1; HRMS (GC-MS, EI) $E$ isomer: calcd for $\mathrm{C}_{12} \mathrm{H}_{14} \mathrm{O}[\mathrm{M}]^{+} 174.1045$, found 174.1028; $Z$ isomer: calcd for $\mathrm{C}_{12} \mathrm{H}_{14} \mathrm{O}[\mathrm{M}]^{+}$174.1045 found 174.1011.

\section{(E)-1-Deuterio-1-phenylbut-1-en-3-one (2b-d).}<smiles>CC(=O)/C=C\c1ccccc1</smiles>

${ }^{1} \mathrm{H}$ NMR $\left(\mathrm{CDCl}_{3}, 300 \mathrm{MHz}\right) \delta 7.50-7.57(\mathrm{~m}, 2.25 \mathrm{H}), 7.35-7.44(\mathrm{~m}, 3 \mathrm{H}), 6.72(\mathrm{t}, J=$ $2.4 \mathrm{~Hz}, 0.75 \mathrm{H}), 2.39(\mathrm{~s}, 3 \mathrm{H}) ;{ }^{2} \mathrm{H} \mathrm{NMR}\left(\mathrm{CDCl}_{3}, 61 \mathrm{MHz}\right) \delta 7.35-7.68(\mathrm{~m}, 0.75 \mathrm{D})$, 6.60-6.85 (m, 0.25D).

\section{Kinetic Resolution of Secondary Propargylic Alcohols}

General Procedure 2 (Table 4, entry 3). Under an Ar atmosphere, AgOTf (6.4 mg, $0.025 \mathrm{mmol})$ and $[\mathrm{Rh}(\mathrm{cod}) \mathrm{Cl}]_{2}(6.2 \mathrm{mg}, 0.0125 \mathrm{mmol})$ were dissolved in acetone $(1.0$ $\mathrm{mL})$ and the mixture was stirred at $\mathrm{rt}$ for $5 \mathrm{~min}$. An acetone $(0.5 \mathrm{~mL})$ solution of $(R)$ BINAP (15.6 mg, $0.025 \mathrm{mmol}$ ) was added and the mixture was stirred at $\mathrm{rt}$ for $5 \mathrm{~min}$. The resulting solution was concentrated to dryness and dissolved in $\mathrm{CH}_{2} \mathrm{Cl}_{2}(0.5 \mathrm{~mL})$. $\mathrm{H}_{2}$ was introduced to the resulting solution in a Schlenk tube. After stirring at $\mathrm{rt}$ for 0.5 $\mathrm{h}$, the resulting solution was concentrated to dryness and dissolved in $\mathrm{CH}_{2} \mathrm{Cl}_{2}(1.0 \mathrm{~mL})$. To this solution was added a $\mathrm{CH}_{2} \mathrm{Cl}_{2}(0.5 \mathrm{~mL})$ solution of 1-phenylhept-1-yn-3-ol (1c, $94.1 \mathrm{mg}, 0.500 \mathrm{mmol})$, and washed remaining substrate away by using $\mathrm{CH}_{2} \mathrm{Cl}_{2}(0.5 \mathrm{~mL})$. The solution was stirred at $25{ }^{\circ} \mathrm{C}$ for $14 \mathrm{~h}$. The conversion of the reaction was determined to be $59.8 \%$ by ${ }^{1} \mathrm{H}$ NMR using 1,4-dimethoxybenzene as an internal standard. The solution was concentrated, and purified by preparative TLC (hexanes:EtOAc = 4:1), which furnished $(E)$-1-phenylhept-1-en-3-one $(\mathbf{2 c}, 50.1 \mathrm{mg}$, $0.270 \mathrm{mmol}, 53 \%$ isolated yield) and (+)-1-phenylhept-1-yn-3-ol [(+)-1c, $33.8 \mathrm{mg}$, $0.180 \mathrm{mmol}, 36 \%$ isolated yield, $[\alpha]_{\mathrm{D}}^{25}=+1.29^{\circ}\left(\mathrm{CHCl}_{3}, c=1.69,81.5 \%\right.$ ee $\left.)\right]$. Selectivity factor $=8.2$. CHIRALPAK OD-H, hexane:2-PrOH $=95: 5,1.0 \mathrm{~mL} / \mathrm{min}$, retention times: $8.3 \mathrm{~min}$ (minor isomer) and $27.7 \mathrm{~min}$ (major isomer).

Table 4, entry 1. $(S)-4-P h e n y l b u t-3-y n-2-o l\left[(S)-1 \mathbf{b},[\alpha]^{25}=-19.6^{\circ}\left(\mathrm{Et}_{2} \mathrm{O}, c\right.\right.$ 1.04, $42.4 \%$ ee); lit. $^{2}[\alpha]^{25}{ }_{D}=-33.9^{\circ}\left(\mathrm{Et}_{2} \mathrm{O}, c 1.1,97 \%\right.$ ee $\left.)\right]$ was obtained for $14 \mathrm{~h}$ at $57.5 \%$ conversion. Selectivity factor $=2.8$. CHIRALPAK OD-H, hexane:2-PrOH $=90: 10,1.0$ $\mathrm{mL} / \mathrm{min}$, retention times: $7.2 \mathrm{~min}$ (minor isomer) and $14.4 \mathrm{~min}$ (major isomer).

Table 4, entry 2. $(S)-1-$ Phenylpent-1-yn-3-ol $\left[(S)-\mathbf{1 a},[\alpha]_{\mathrm{D}}^{25}=-15.0^{\circ}\left(\mathrm{Et}_{2} \mathrm{O}, c\right.\right.$ 1.49, $76.0 \%$ ee); lit. ${ }^{1}[\alpha]^{25}{ }_{\mathrm{D}}=+19.2^{\circ}\left(\mathrm{Et}_{2} \mathrm{O}, c 1.24,94 \%\right.$ ee $\left.)\right]$ was obtained for $72 \mathrm{~h}$ at $58.5 \%$ conversion. Selectivity factor $=7.3$. CHIRALPAK OD-H, hexane:2-PrOH $=95: 5,1.0$ $\mathrm{mL} / \mathrm{min}$, retention times: $8.8 \mathrm{~min}$ (minor isomer) and $24.4 \mathrm{~min}$ (major isomer).

Table 4, entry 4. $(S)$-4-Methyl-1-phenylpent-1-yn-3-ol $\left[(S)-1 \mathbf{d},[\alpha]_{\mathrm{D}}^{25}=-0.28^{\circ}\right.$ 
$\left(\mathrm{CHCl}_{3}, c 1.72,58.1 \%\right.$ ee $)$; lit. ${ }^{3}[\alpha]^{25}{ }_{\mathrm{D}}=+3.2^{\circ}\left(\mathrm{CHCl}_{3}, c 6.8,90 \%\right.$ ee $\left.)\right]$ was obtained for $28 \mathrm{~h}$ at $51.2 \%$ conversion. Selectivity factor $=6.1$. CHIRALPAK OD-H, hexane:2$\mathrm{PrOH}=95: 5,1.0 \mathrm{~mL} / \mathrm{min}$, retention times: $7.6 \mathrm{~min}$ (minor isomer) and $17.2 \mathrm{~min}$ (major isomer).

Table 4, entry 5. $(S)-4,4-D i m e t h y l-1-p h e n y l p e n t-1-y n-3-o l\left[(S)-1 e,[\alpha]_{D}^{25}=-0.23^{\circ}\right.$ $\left(\mathrm{CHCl}_{3}, c 1.73,61.7 \%\right.$ ee $)$; lit. ${ }^{3}[\alpha]^{25}=+2.4^{\circ}\left(\mathrm{CHCl}_{3}, c 4.0,94 \%\right.$ ee $\left.)\right]$ was obtained for $38 \mathrm{~h}$ at $58.2 \%$ conversion. Selectivity factor $=4.7$. CHIRALPAK OD-H, hexane: $2-$ $\mathrm{PrOH}=95: 5,1.0 \mathrm{~mL} / \mathrm{min}$, retention times: $8.7 \mathrm{~min}$ (minor isomer) and $12.8 \mathrm{~min}$ (major isomer); lit. ${ }^{3}$ CHIRALPAK OD, hexane:2-PrOH $=90: 10$, retention times: 7.4 min $(R$ isomer) and $10.1 \mathrm{~min}(S$ isomer).

Table 4, entry 6. (-)-1-(4-Methoxyphenyl)pent-1-yn-3-ol $\left[(-)-\mathbf{1 f},[\alpha]_{\mathrm{D}}^{25}=-1.15^{\circ}\right.$ $\left(\mathrm{CHCl}_{3}, c 1.74,62.3 \%\right.$ ee)] was obtained for $45 \mathrm{~h}$ at $54.5 \%$ conversion. Selectivity factor $=5.8$. CHIRALPAK OD-H, hexane: $2-\mathrm{PrOH}=95: 5,1.0 \mathrm{~mL} / \mathrm{min}$, retention times: $11.5 \mathrm{~min}$ (minor isomer) and $40.8 \mathrm{~min}$ (major isomer).

Table 4, entry 7. (-)-1-(4-Trifluoromethylphenyl)pent-1-yn-3-ol $\left[(-)-\mathbf{1 g},[\alpha]_{\mathrm{D}}^{25}=\right.$ $-1.05^{\circ}\left(\mathrm{CHCl}_{3}, c 1.76,78.0 \%\right.$ ee $\left.)\right]$ was obtained for $64 \mathrm{~h}$ at $60.0 \%$ conversion. Selectivity factor $=7.2$. CHIRALPAK OD-H, hexane:2-PrOH $=99: 1,1.0 \mathrm{~mL} / \mathrm{min}$, retention times: $19.7 \mathrm{~min}$ (minor isomer) and $22.6 \mathrm{~min}$ (major isomer).

Table 4, entry 8. (-)-1-o-Tolylpent-1-yn-3-ol $\left[(-)-1 \mathbf{h},[\alpha]^{25}=-3.07^{\circ}\left(\mathrm{CHCl}_{3}, c 1.79\right.\right.$, $80.0 \%$ ee)] was obtained for $42 \mathrm{~h}$ at $54.9 \%$ conversion. Selectivity factor $=11.5$. CHIRALPAK OD-H, hexane:2-PrOH = 95:5, $1.0 \mathrm{~mL} / \mathrm{min}$, retention times: $8.9 \mathrm{~min}$ (minor isomer) and $15.8 \mathrm{~min}$ (major isomer).

\section{References}

(1) Gao, G.; Moore, D.; Xie, R.-G.; Pu, L. Org. Lett. 2002, 4, 4143-4146.

(2) Kazmaier, U.; Zumpe, F. L. Eur. J. Org. Chem. 2001, 4067-4076.

(3) Frantz, D. E.; Fässler, R.; Carreira, E. M. J. Am. Chem. Soc. 2000, 122, 1806-1807.

(4) Cassel, J. A.; Leue, S.; Kann, N. C. J. Org. Chem. 2002, 67, 9460-9463.

(5) Yang, D.; Chen, Y.-C.; Zhu, N.-Y. Org. Lett. 2004, 6, 1577-1580.

(6) Arisawa, M.; Torisawa, Y.; Nagasawa, M. J. Org. Chem. 1997, 62, 4327-4329.

(7) Plunian, B.; Mortier, J.; Vaultier, M. J. Org. Chem. 1996, 61, 5206-5207.

(8) Wipf, P.; Rahman, L. T.; Rector, S. R. J. Org. Chem. 1998, 63, 7132-7133.

(9) Ooi, T.; Ohara, D.; Tamaru, M.; Maruoka, K. J. Am. Chem. Soc. 2004, 126, 68446845.

(10) Okano, T.; Ohno, K.; Kiji, J. Chem. Lett. 1996, 1041-1042. 

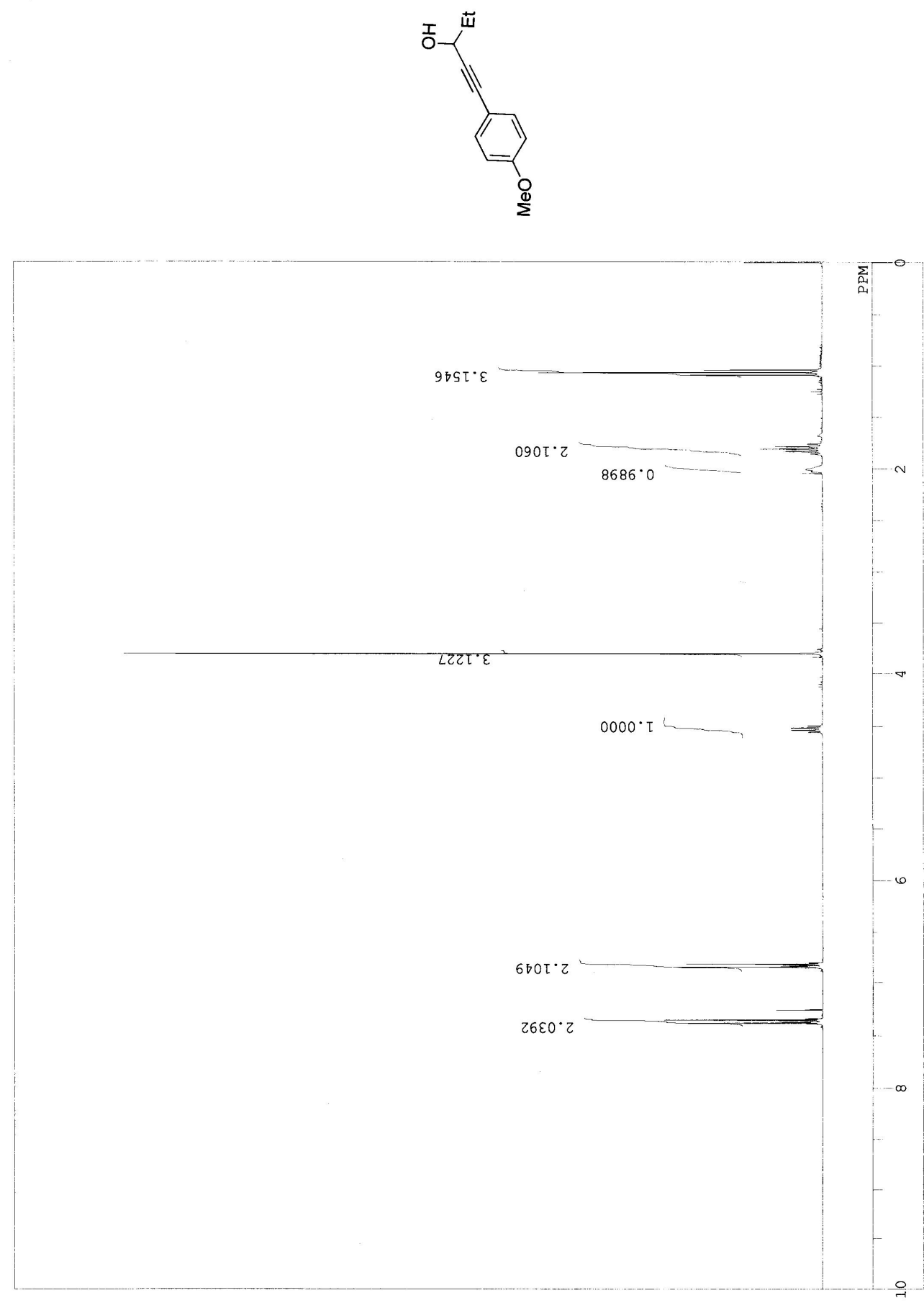


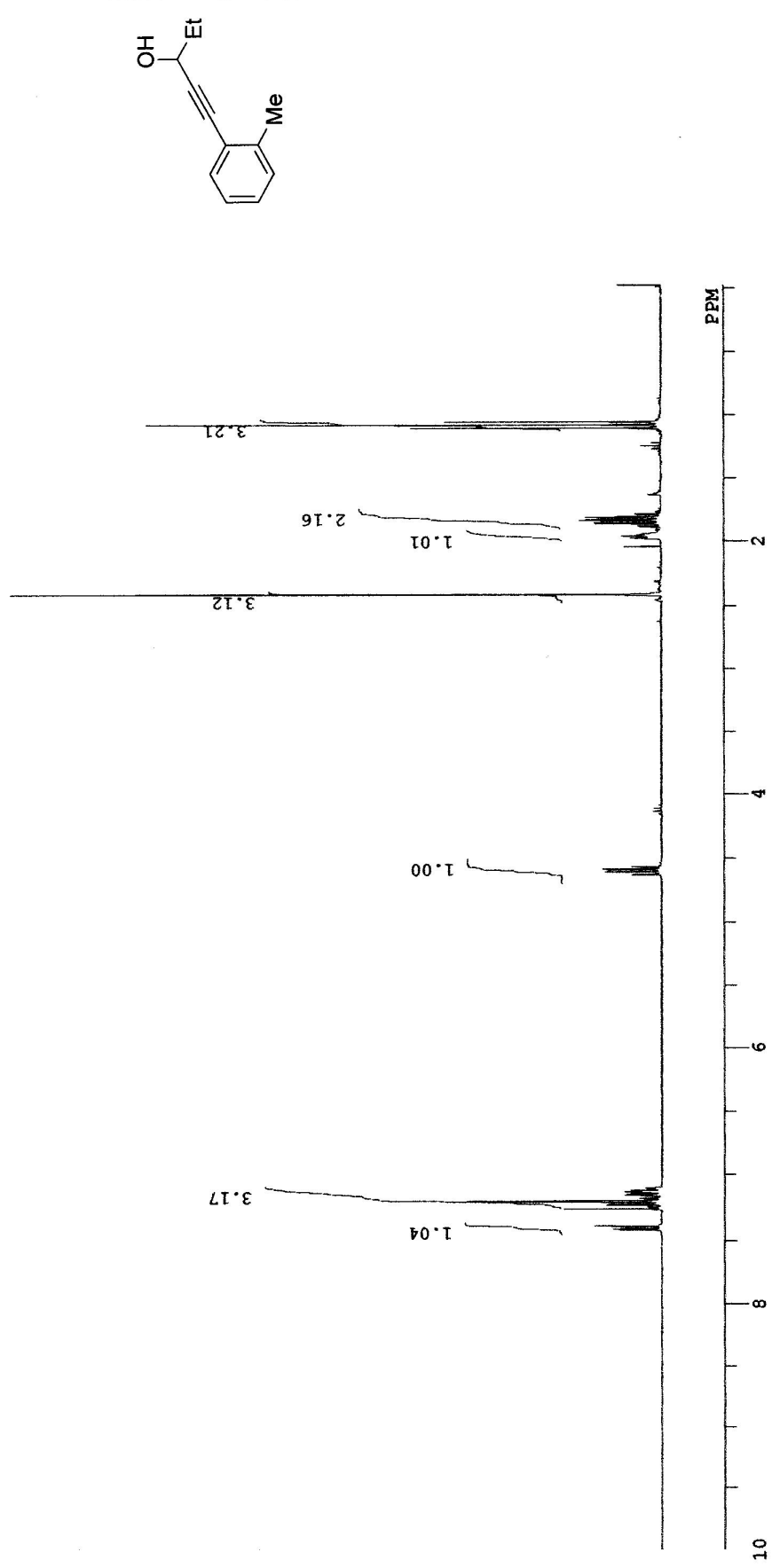




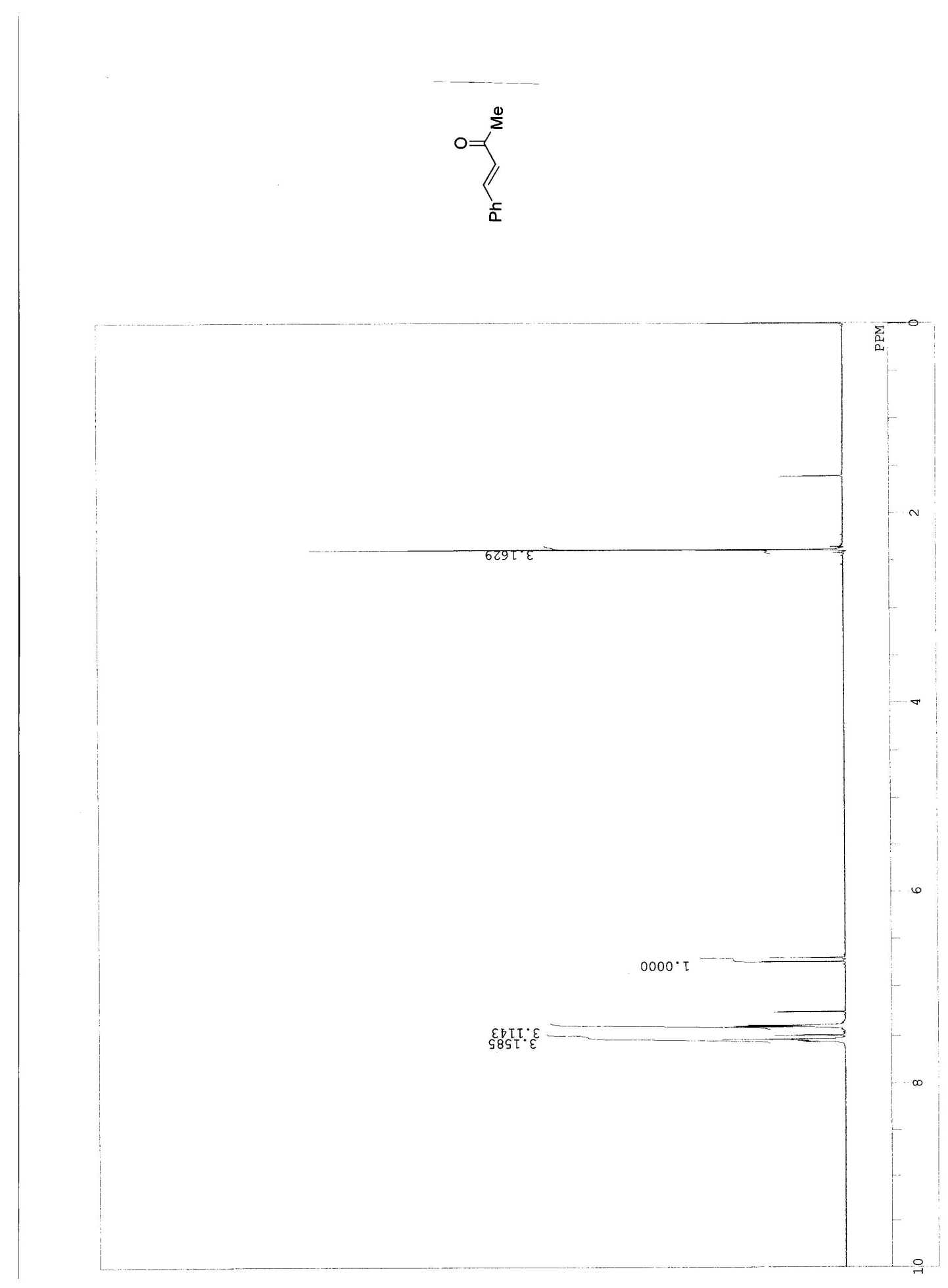




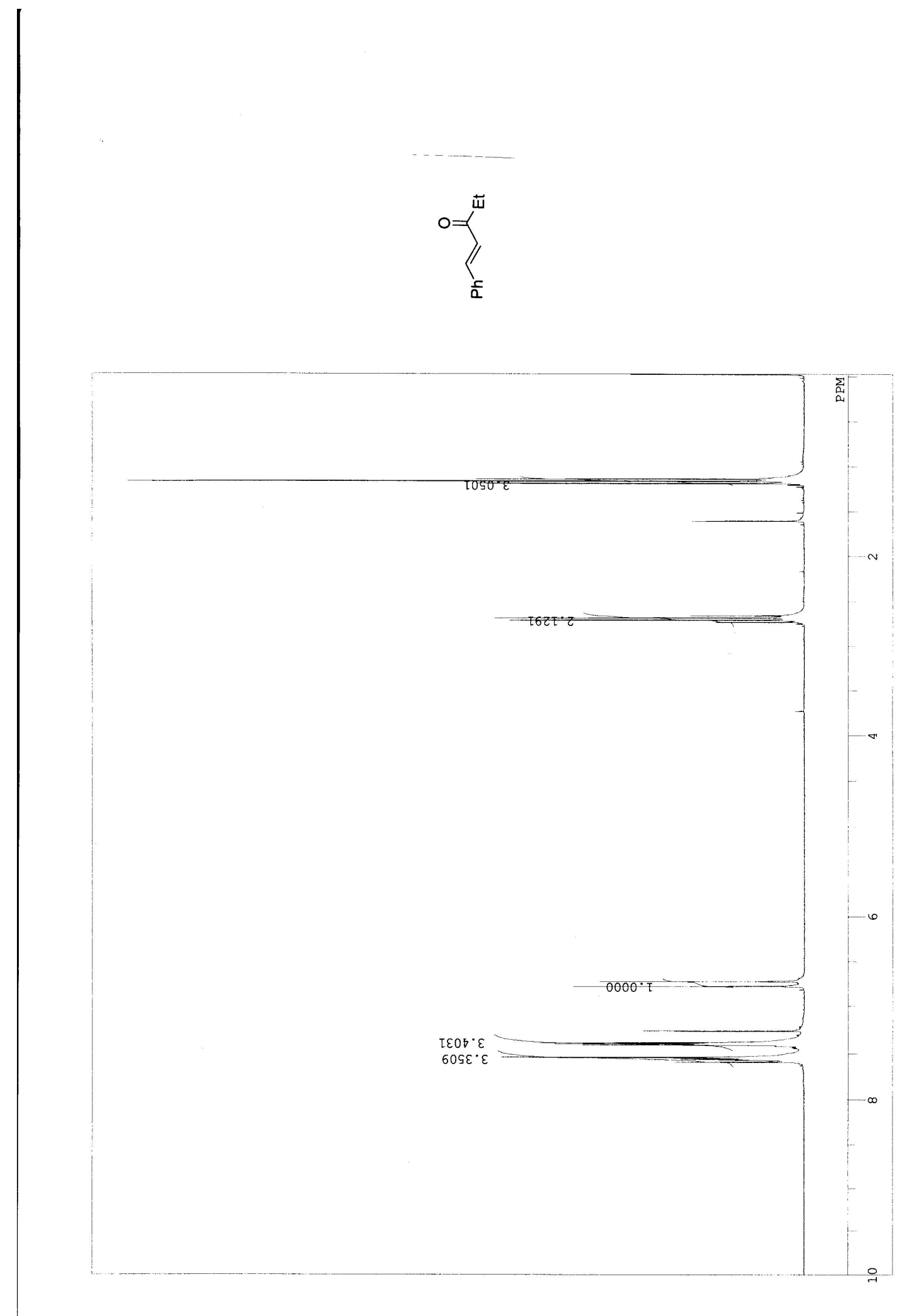



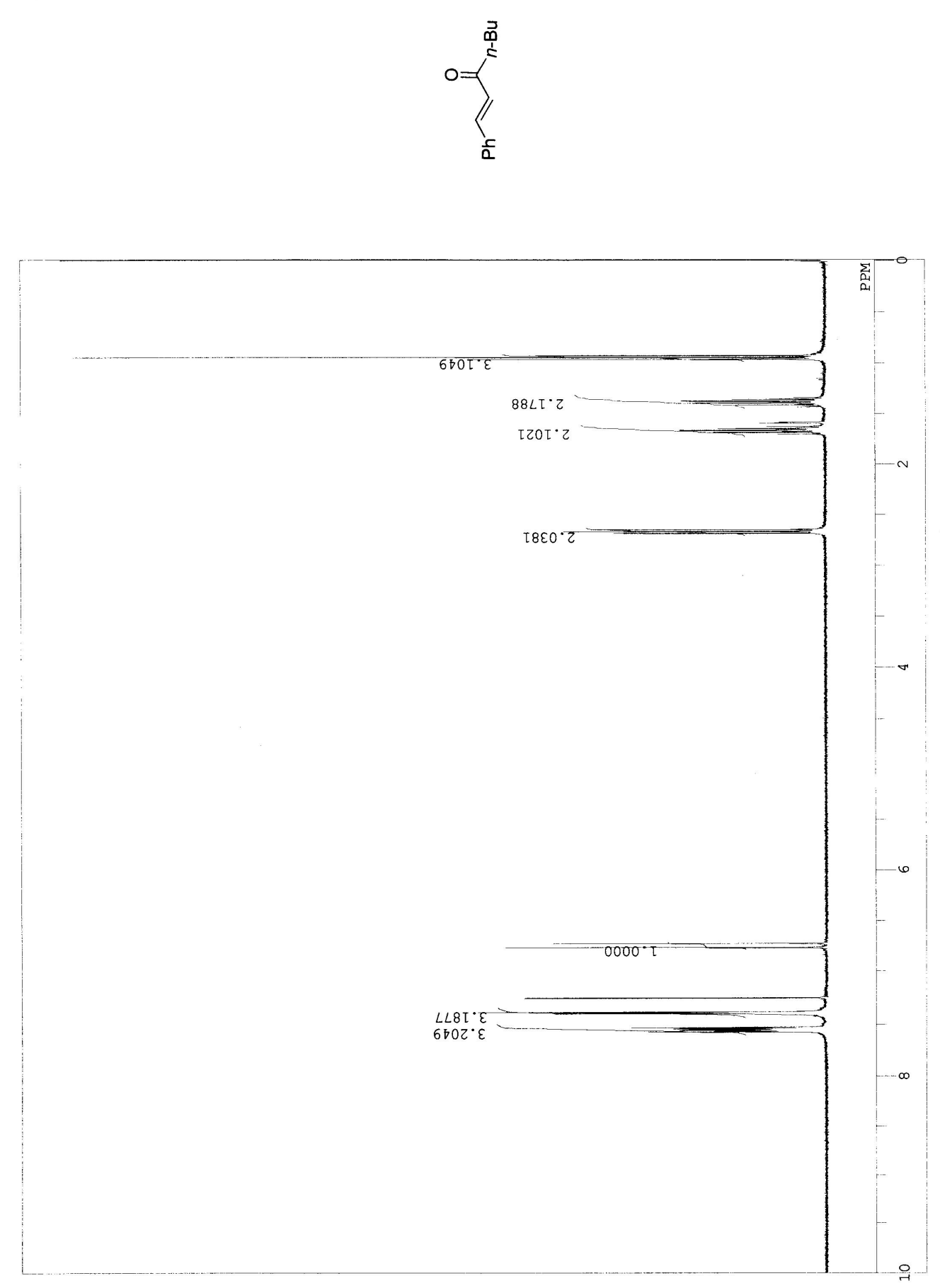


$$
0=\sum_{\frac{1}{a}}^{\frac{a}{1}}
$$

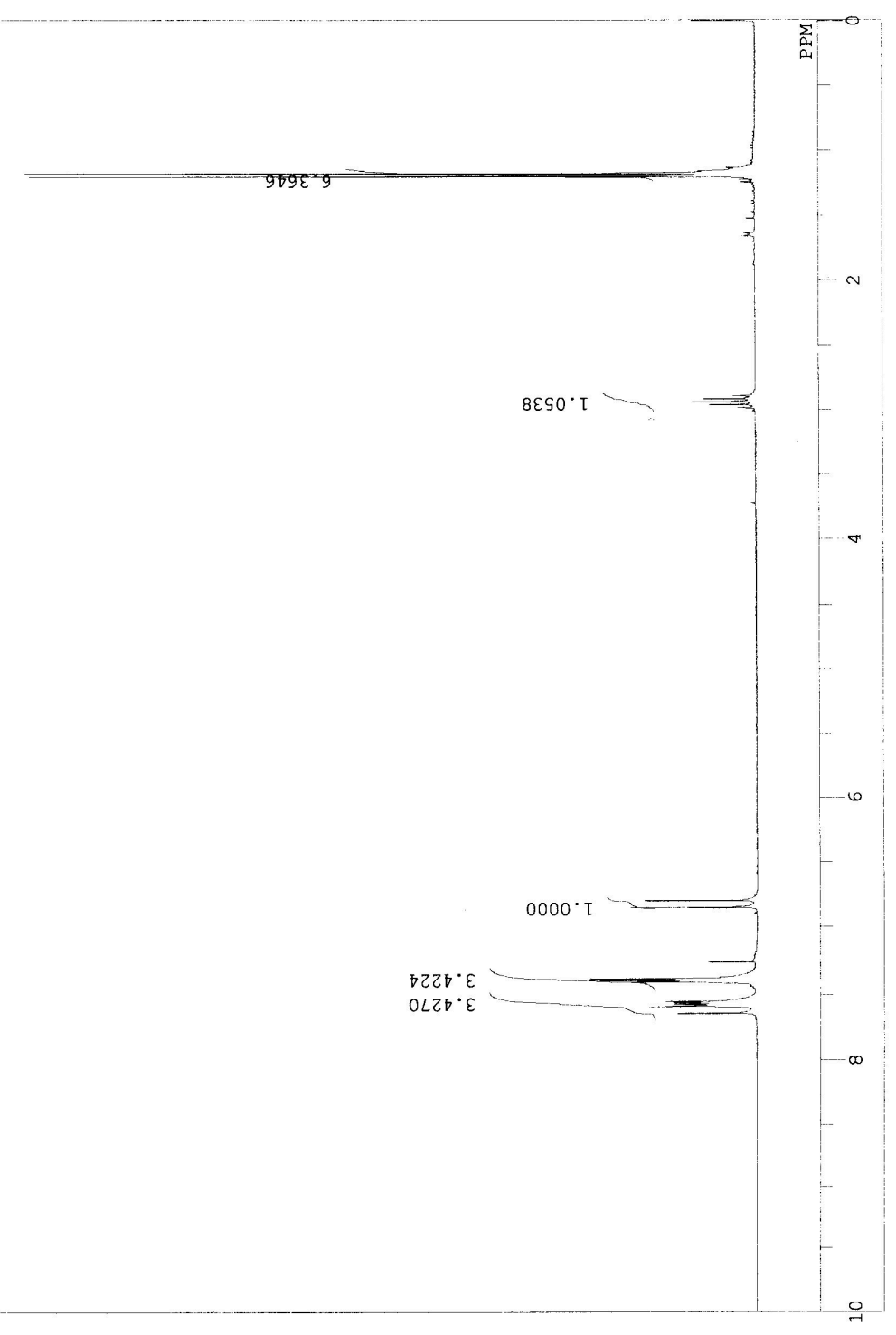


$=\sum_{\frac{c}{0}}^{\frac{3}{9}}$

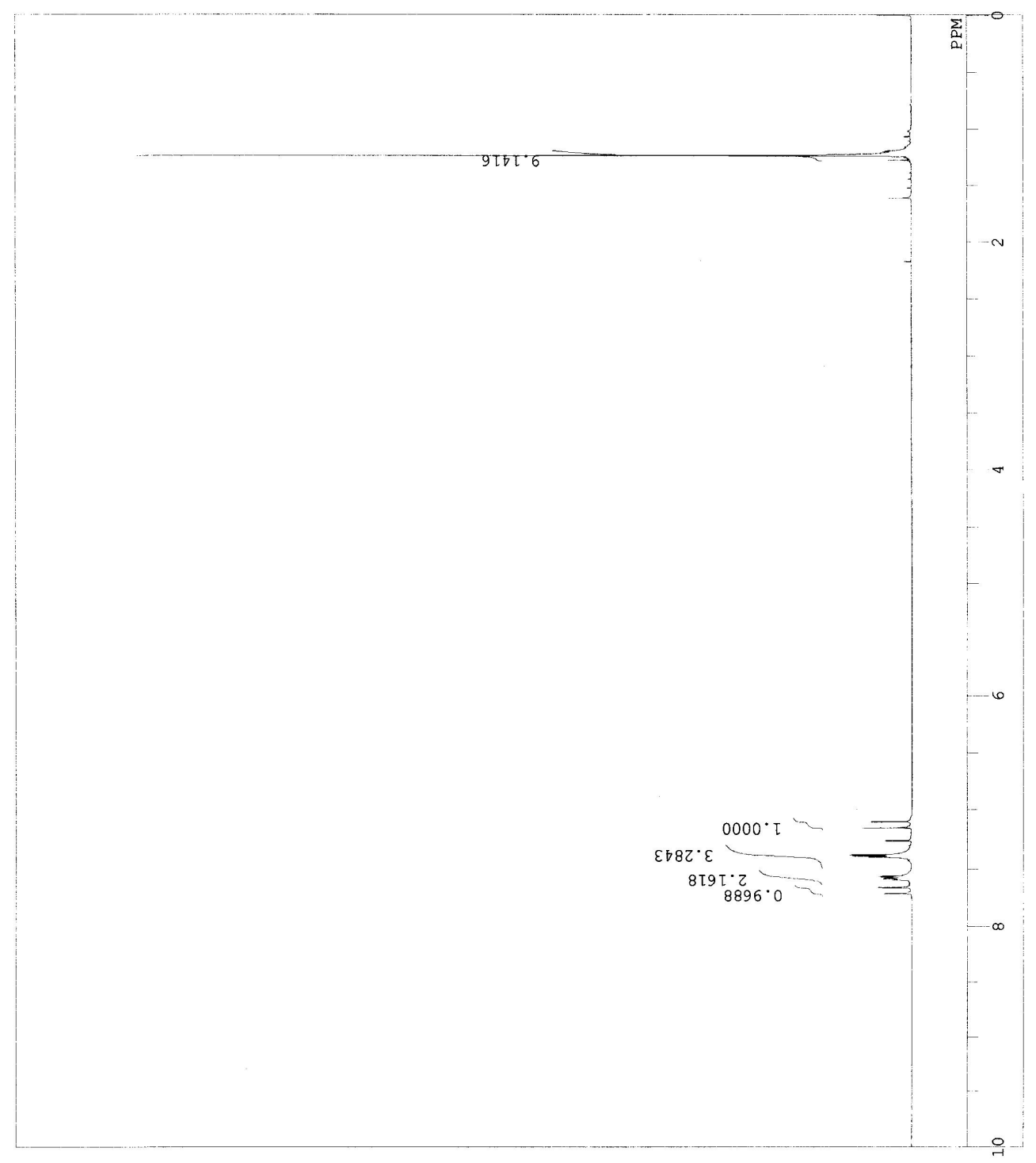




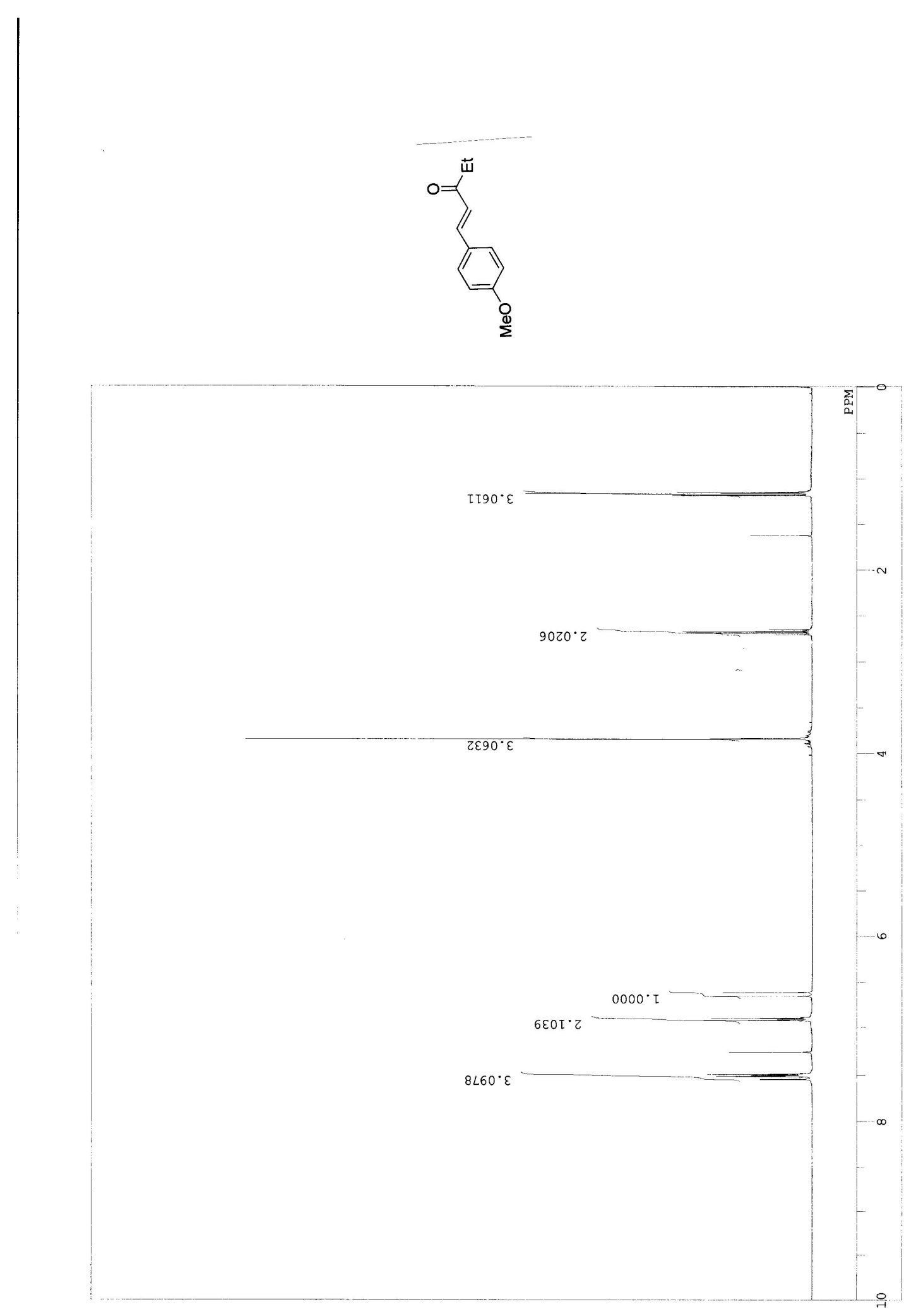



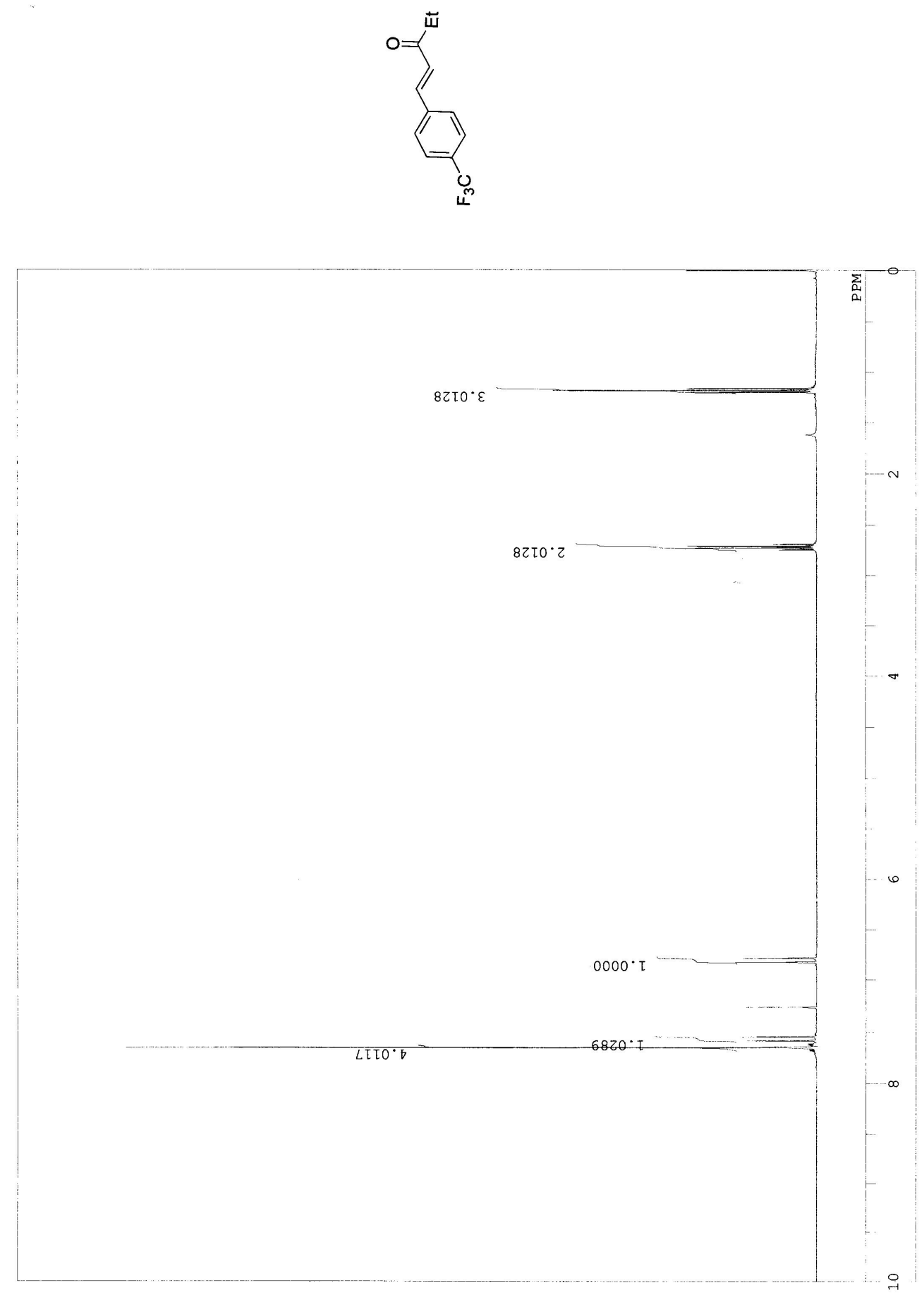


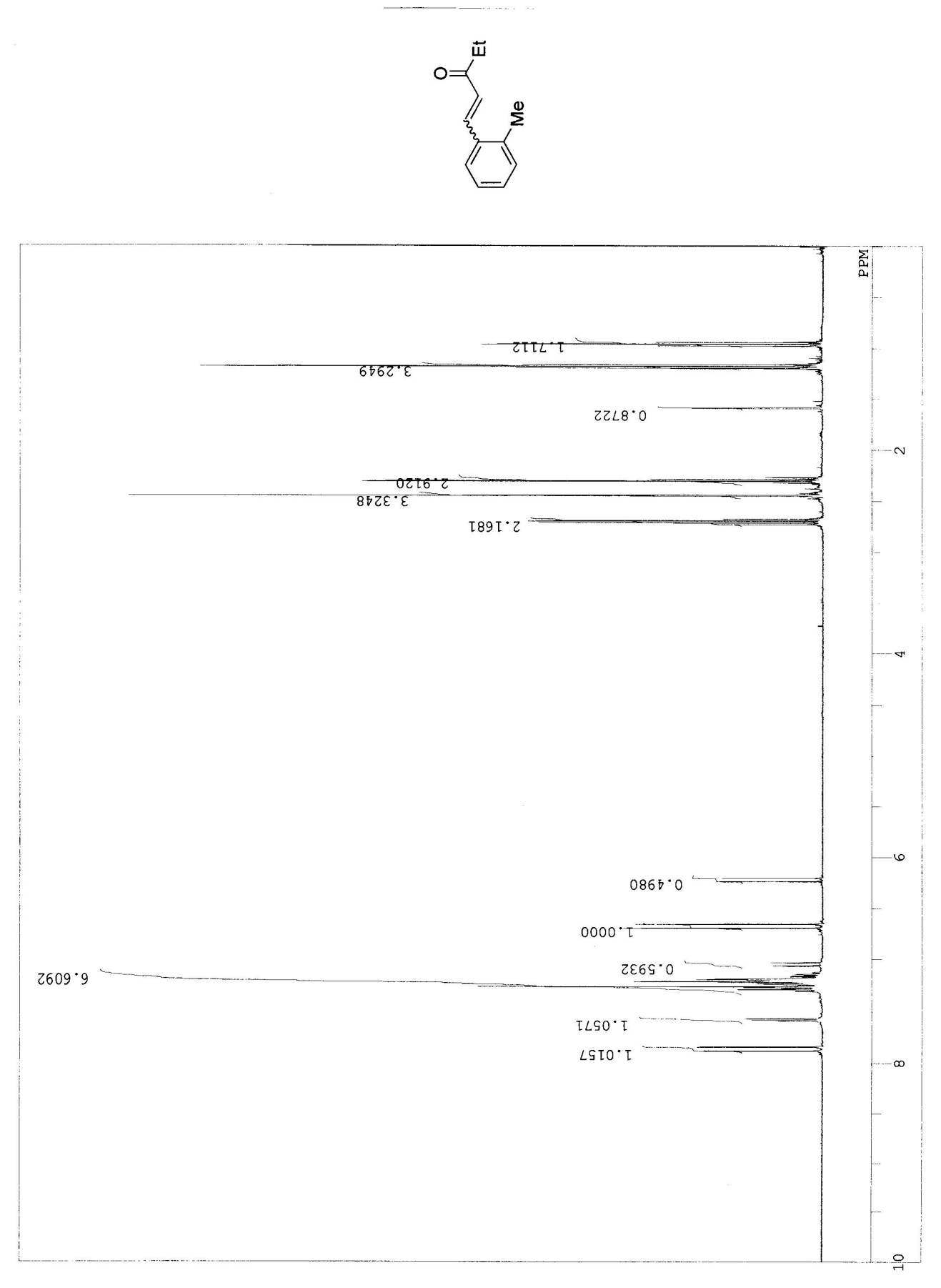

